\title{
Multiple Periodic Solutions for Some Classes of First-Order Hamiltonian Systems
}

\author{
Mohsen Timoumi \\ Department of Mathematics, Faculty of Sciences, Monastir, Tunisia \\ E-mail: Mohsen.Timoumi@fsm.rnu.tn \\ Received April 15, 2011; revised May 19, 2011; accepted May 22, 2011
}

\begin{abstract}
Considering a decomposition $\mathbb{R}^{2} N=A \oplus B$ of $\mathbb{R}^{2} N$, we prove in this work, the existence of at least $(1+\operatorname{dim} A)$ geometrically distinct periodic solutions for the first-order Hamiltonian system $J x^{\prime}(t)+H^{\prime}(t, x(t))+e(t)=0$ when the Hamiltonian $H(t, u+v)$ is periodic in $(t, u)$ and its growth at infinity in $v$ is at most like or faster than $|v|^{a}, 0 \leq a<1$, and $e$ is a forcing term. For the proof, we use the Least Action Principle and a Generalized Saddle Point Theorem.
\end{abstract}

Keywords: Hamiltonian Systems, Partial Nonlinearity, Multiple Periodic Solutions, Critical Point Theory

\section{Introduction}

Consider the nonautonomous first-order Hamiltonian system

$$
J x^{\prime}(t)+H^{\prime}(t, x(t))+e(t)=0
$$

where $H: \mathbb{R} \times \mathbb{R}^{2 N} \rightarrow \mathbb{R}, \mathbb{R},(t, x) \rightarrow H(t, x)$ is a continuous function, $T-$ periodic $(T>0)$ in the first variable and differentiable with respect to the second variable with continuous derivative $H^{\prime}(t, x)=\frac{\partial H}{\partial x}(t, x)$, $e: \mathbb{R} \rightarrow \mathbb{R}^{2} N$ is a continuous $T$-periodic function with mean value zero and $\mathrm{J}$ is the standard symplectic matrix:

$$
J=\left(\begin{array}{cc}
0 & -I_{N} \\
I_{N} & 0
\end{array}\right)
$$

$I_{N}$ being the identity matrix of order $N$.

Using variational methods, there have been many papers devoted to the existence of periodic solutions for $(\mathcal{H})$, we refer the readers to [1-5] and the references therein. However, there are few papers discussing the multiplicity of periodic solutions for $(\mathcal{H})$ (see [6-9]). Under the assumptions that $\mathrm{H}$ is periodic in $x_{1}, \cdots, x_{p}$, where $1 \leq p \leq 2 N-1, \quad x=\left(x_{1}, \cdots, x_{2 N}\right)$ and there exists $f \in L^{2}\left(0, T ; \mathbb{R}^{+}\right)$such that

$$
\begin{gathered}
\left|H^{\prime}(t, x)\right| \leq f(t), \forall x \in \mathbb{R}^{2 N}, \quad \text { a.e.t } \in[0,1] . \\
\int_{0}^{T} H(t, x) \mathrm{d} t \rightarrow \pm \infty \text { as }|x| \rightarrow \infty, x \in\{0\} \times \mathbb{R}^{2 N-p},
\end{gathered}
$$

the author has shown in [9] that system $(\mathcal{H})$ possesses at least $(p+1)$ geometrically distinct periodic solutions. The first goal of this note is to generalize the existence result of multiple periodic solutions obtained above to the sublinear case. Precisely, consider a decomposition $\mathbb{R}^{2 N}=A \oplus B$ of $\mathbb{R}^{2 N}$ with

$$
A=\operatorname{space}\left\{e_{1}, \cdots, e_{i_{p}}\right\}, B=\operatorname{space}\left\{e_{i_{p+1}}, \cdots, e_{i_{2 N}}\right\}
$$

where $0 \leq p \leq 2 N-1$ and $\left(e_{i}\right)_{1 \leq i \leq 2 N}$ is the standard basis of $\mathbb{R}^{2 N}$ and let us denote $P_{A}$ (resp. $P_{B}$ ) the projection of $\mathbb{R}^{2 N}$ into $A$ (resp. $B$ ). We obtain the following result

Theorem 1.1 Assume that $H$ satisfies $\left(H_{0}\right) H$ is periodic in the variables. $x_{i_{1}}, \cdots, x_{i_{p}}$;

$\left(H_{1}\right)$ There exist $\alpha \in[0,1]$ and two $T$-periodic functions $a \in L \frac{1}{1-\alpha}\left(0, T ; \mathbb{R}^{+}\right)$and $b \in L^{2}\left(0, T ; \mathbb{R}^{+}\right)$such that

$\left|H^{\prime}(t, x)\right| \leq a(t)\left|P_{B}(x)\right|^{\alpha}+b(t), \forall x \in \mathbb{R}^{2 N}, \quad$ a.e.t $\in[0,1]$,

$\left(\mathrm{H}_{2}\right)$ Either

1)

$$
\frac{1}{|x|^{2 \alpha}} \int_{0}^{T} H(t, x) \mathrm{d} t \rightarrow \infty \text { as }|x| \rightarrow \infty, x \in B
$$

or

$$
\frac{1}{|x|^{2 \alpha}} \int_{0}^{T} H(t, x) \mathrm{d} t \rightarrow-\infty \text { as }|x| \rightarrow \infty, x \in B
$$

Then the Hamiltonian system $(\mathcal{H})$ possesses at least $(p+1) T-$ periodic solutions geometrically distinct. 
Example 1.1 Let $a: \mathbb{R}^{2 N} \rightarrow \mathbb{R}$ be a periodic and continuously differentiable function. Consider the Hamiltonian:

$$
H(t, r, p)=\left(\frac{1}{2}+\sin \left(\frac{2 \pi}{T} t\right)\right)|p-a(r)|^{\frac{3}{2}}
$$

Then $H$ satisfies conditions $\left(H_{0}\right)-\left(H_{2}\right)$ with $A=\mathbb{R}^{N} \times 0$ and $B=\{0\} \times \mathbb{R}^{N}$.

It is easy to see that conditions $\left(H_{1}\right),\left(H_{2}\right)$ don't cover some sublinear cases like

$$
\begin{aligned}
& H(t, r, p)=\left(\frac{1}{2}+\cos \left(\frac{2 \pi}{T} t\right)\right) \frac{|p-a(r)|^{2}}{\ln \left(2+|p-a(r)|^{2}\right)}, \\
& \forall(t, r, p) \in \mathbb{R} \times \mathbb{R}^{2 N}
\end{aligned}
$$

The second goal of this paper is to study the existence of multiple periodic solutions for $(\mathcal{H})$ when the Hamiltonian $H$ satisfies a nonlinearity condition which covers the cases like (1.4). Precisely, we will require the nonlinearity to have a partial growth at infinity faster than $|x|^{\alpha}, 0 \leq \alpha<1$

Our second main result is:

Theorem 1.2 Consider a nonincreasing positive function $\omega \in C\left(\left[0,+\infty\left[, \mathbb{R}^{+}\right)\right.\right.$with the properties:

$$
\begin{gathered}
\liminf _{s} \rightarrow+\infty \frac{\omega(s)}{\omega(s)}>0, \\
\omega(s) \rightarrow 0, \omega(s) s \rightarrow+\infty \text { as } s \rightarrow+\infty,
\end{gathered}
$$

and assume that $H$ atisfies $\left(H_{0}\right)$ and the following assumptions

$\left(H_{3}\right)$ There exist a positive constant $a \mathrm{~d}$ a function $g \in L^{2}\left(0, T ; \mathbb{R}^{+}\right)$such that for all $\forall x \in \mathbb{R}^{2 N}$ and a.e.t $\in[0,1]$

$$
\left|H^{\prime}(t, x)\right| \leq a \omega\left(\left|P_{B}(x)\right|\right)\left|P_{B}(x)\right|+g(t),
$$

$\left(\mathrm{H}_{4}\right)$ Either

1) $\frac{1}{\left\lceil\left.\omega(|x|)|x|\right|^{2}\right.} \int_{0}^{T} H(t, x) \mathrm{d} t \rightarrow+\infty$ as $|x| \rightarrow \infty, x \in B$,

2) $\frac{1}{\left[\left.\omega(|x|)|x|\right|^{2}\right.} \int_{0}^{T} H(t, x) \mathrm{d} t \rightarrow-\infty$ as $|x| \rightarrow \infty, x \in B$.

Then the system $(\mathcal{H})$ possesses at least $(p+1)$ geo-metrically distinct $T$-periodic solutions.

Remark 1.1 The Hamiltonian $H$ defined in (1.4) satisfies the conditions $\left(H_{3}\right),\left(H_{4}\right)$ introduced above with $\omega(s)=\frac{1}{\ln \left(2+s^{2}\right)}, s \geq 0$,

\section{Preliminaries}

Firstly, let us recall a critical point theorem due to G.
Fournier, D. Lupo, M. Ramos and M. Willem [10]. Given a Banach space $\mathrm{E}$ and a complete connected Finsler manifold $\mathrm{V}$ of class $C^{2}$, we consider the space $X=E x V$. Let $E=W \oplus Z$ (topological direct sum) and $\left(E_{n} \oplus Z_{n}\right)$ be a sequence of closed subspaces with $Z_{n} \subset Z, W_{n} \subset W, 1 \leq \operatorname{dim} W_{n}<\infty$. Define $X_{n}=E_{n} x V$. For $f \in C^{1}(X, \mathbb{R})$, we denote by $f_{n}=f_{/ X_{n}}$. Then we have $f_{n} \in C^{1}\left(X_{n}, \mathbb{R}\right)$ for all $n \geq 1$.

Definition 2.1 Let $\in C^{1}(X, \mathbb{R})$. The function $f$ satisfies the Palais-Smale condition with respect to $\left(X_{n}\right)$ at a level $c \in \mathbb{R}$ if every sequence $\left(X_{n}\right)$ satisfying

$$
x_{n} \in X_{n}, f\left(x_{n}\right) \rightarrow c, f_{n}^{\prime}\left(x_{n}\right) \rightarrow 0
$$

has a subsequence which converges in $X$ to a critical point of $f$. The above property will be referred as the $(P S)_{c}^{*}$ condition with respect to $\left(X_{n}\right)$.

Theorem 2.1 (Generalized Saddle Point Theorem). Assume that there exist constants $r>0$ and $\alpha<\beta \leq \gamma$ such that

1) $f$ satisfies the $(P S)^{*}$ condition with respect to $\left(X_{n}\right)$ for every $c \in[\beta, \gamma]$,

2) $f(w, v) \leq \alpha$ for every $(w, v) \in W \times V$ such that $\|w\|=r$,

3) $f(z, v) \geq \beta$ for every $(z, v) \in Z \times V$,

4) $f(w, v) \leq \gamma$ for every $(w, v) \in W \times V$ such that $\|w\| \leq r$.

Then $f^{-1}([\beta, \gamma])$ contains at least cuplength $(V)+1$ critical points of $f$.

Consider the Hilbert space $E=H^{\frac{1}{2}}\left(S^{1}, \mathbb{R}^{2 N}\right)$ where $\mathbb{R} /(T \mathbb{Z})$ and the continuous quadratic form $Q$ efined in E y

$$
Q(x)=\frac{1}{2} \int_{0}^{T} J x^{\prime}(t) \cdot x(t) \mathrm{d} t
$$

where $x, y$ inside the sign integral is the inner product of $x, y \in \mathbb{R}^{2 N}$. Let us denote by $E^{0}, E^{-}, E^{+}$respectively the subspaces of $E$ on which $Q$ is null, negative definite and positive definite. It is well known that these sub-spaces are mutually orthogonal in $L^{2}\left(S^{1}, \mathbb{R}^{2 N}\right)$ and in $E$ with respect to the bilinear form:

$$
B(x, y)=\frac{1}{2} \int_{0}^{T} J x^{\prime}(t) \cdot y(t) \mathrm{d} t, \quad x, y \in E
$$

associated to $Q$. If $x \in E^{+}$and $y \in E^{-}$then $B(x, y)=0$ and $Q(x+y)=Q(x)+Q(y)$.

For $x=x^{+}+x^{-}+x^{0} \in E$, the expression

$$
\|x\|=\left[Q\left(x^{+}\right)+Q\left(x^{-}\right)+\left|x^{0}\right|^{2}\right]^{\frac{1}{2}}
$$

is an equivalent norm in E. Moreover, the space $E$ is compactly embedded in $L^{2}\left(S^{1}, \mathbb{R}^{2 N}\right)$ for all $s \in[1, \infty]$. In particular for all $s \in[1, \infty]$, there exists a constant $\lambda_{s}>0$ such that for all $x \in E$, 


$$
\|x\|_{L^{s}} \leq \lambda_{s}\|x\| .
$$

\section{Proof of the Theorems}

Firstly, let us remark that if $x(t)$ is a periodic solution of $(\mathcal{H})$, then by replacing $t$ by $-t$ in $(\mathcal{H})$ we obtain

$$
J x^{\prime}(-t)+H^{\prime}(-t, x(-t))+e(-t)=0 .
$$

So it is clear that the function $y(t)=x(-t)$ is a periodc solution of the system

$$
J y^{\prime}(t)-H^{\prime}(-t, y(-t))-e(-t)=0 .
$$

Moreover, $-H(-t, x)$ satisfies $\left(H_{2}\right)(i)$ (resp. $\left(H_{4}\right)(i)$ ) whenever $H(t, x)$ satisfies $\left(H_{2}\right)(i i)$ (resp. $\left.\left(H_{4}\right)(i i)\right)$. Hence, in the following, we will assume that $H$ satisfies $\left(H_{2}\right)(i)$ in Theorem 1.1 and $\left(H_{4}\right)(i)$ in Theorem 1.2.

Associate to the system $(\mathcal{H})$ the functional $\varphi$ defined on the space $E$, by:

$$
\varphi(u)=\frac{1}{2} \int_{0}^{T} J u^{\prime}(t) \cdot u(t) \mathrm{d} t+\int_{0}^{T}(H(t, u)+e(t) \cdot u(t)) \mathrm{d} t .
$$

It is well known that the functional $\varphi$ is continuously differentiable in $E$ and critical points of $\varphi$ on $E$ corres-pond to the $T$ - periodic solutions of the system $(\mathcal{H})$, moreover one has

$$
\varphi^{\prime}(u) v=\int_{0}^{T}\left[J u^{\prime}(t)+H^{\prime}(t, u(t))+e(t)\right] \cdot v(t) \mathrm{d} t
$$

for all $u, v \in E$. Consider the subspaces $W=E^{-}$, $Z=E^{+} \oplus B$ of $E$ and the quotient space

$$
V=A /\left\{x \sim x+e_{i}, i=i_{1}, \cdots, i_{p}\right\}
$$

which is nothing but the torus $T_{p}$. We regard the functional $\varphi$ as defined on the space $X=(W \oplus Z) \times V$ as follows

$$
\begin{aligned}
\varphi(u+v) & =\frac{1}{2} \int_{0}^{T} J u^{\prime}(t) \cdot u(t) \mathrm{d} t+\int_{0}^{T} H(t, u(t)+v(t)) \mathrm{d} t \\
& +\int_{0}^{T} e(t) \cdot u(t) \mathrm{d} t
\end{aligned}
$$

To find critical points of $\varphi$ we will apply Theorem 2.1 to this functional with respect to the sequence of subspaces $X_{n}=E_{n} \times V$, where for $n \geq 0$

$$
E_{n}=\left\{x \in E: x(t)=\sum_{|m| \leq n} \exp \left(\frac{2 \pi}{T} m t J\right) \hat{u}_{m} \text { a.e. }\right\} .
$$

Proof of the Theorem 1.1. Assume $\left(H_{0}\right),\left(H_{1}\right)$ and $\left(H_{2}\right)(i)$ hold. Firstly, let us check the PalaisSmale condition.

Lemma 2.1. For all level $c \in \mathbb{R}$, the functional $\varphi$ satisfies the $(P S)_{c}^{*}$ condition with respect to the sequence $\left(X_{n}\right)_{n \in N}$.
Proof. Let $c \in \mathbb{R}$ and let $\left(u_{n}, v_{n}\right)_{n \in \mathbb{N}}$ be a sequence of X such that for all $n \in \mathbb{N},\left(u_{n}, v_{n}\right) \in X_{n}$ and

$$
\varphi\left(u_{n}+v_{n}\right) \rightarrow c \text { and } \varphi_{n}^{\prime}\left(u_{n}+v_{n}\right) \rightarrow 0 \text { as } n \rightarrow \infty, \text { (3.1) }
$$

where $\varphi_{n}$ is the functional $\varphi$ restricted to $X_{n}$. Set $u_{n}=u_{n}^{+}+u_{n}^{-}+u_{n}^{0}$ with $u_{n}^{+} \in E^{+}, u_{n}^{-} \in E^{+}, u_{n}^{0} \in B$. We have the relation

$$
\begin{aligned}
& \varphi_{n}^{\prime}\left(u_{n}+v_{n}\right) u_{n}^{+} \\
& =\left\|u_{n}^{+}\right\|^{2}+\int_{0}^{T}\left[H^{\prime}\left(t, u_{n}+v_{n}\right)+e(t)\right] \cdot u_{n}^{+} \mathrm{d} t
\end{aligned}
$$

Since $\varphi_{n}^{\prime}\left(u_{n}+v_{n}\right) \rightarrow 0$ as $n \rightarrow \infty$, there exists a constant $c_{1}>0$ such that

$$
\forall n \in N,\left|\varphi_{n}^{\prime}\left(u_{n}+v_{n}\right) u_{n}^{+}\right| \leq c_{1}\left\|u_{n}^{+}\right\| .
$$

By assumption $\left(H_{1}\right)$ and Hölder's inequality, with $p=\frac{1}{\alpha}, q=\frac{1}{1-\alpha}$, we have

$$
\begin{aligned}
& \left|\int_{0}^{T} H^{\prime}\left(t, u_{n}+v_{n}\right) \cdot u_{n}^{+} \mathrm{d} t\right| \\
& \leq \int_{0}^{T}\left[a(t)\left|P_{B}\left(u_{n}(t)\right)\right|^{\alpha}+b(t)\right]\left|u_{n}^{+}\right| \mathrm{d} t \\
& \leq\left\|u_{n}^{+}\right\|_{L^{2}}\left[\|a\|_{L^{1-\alpha}} \frac{2}{\|}\left\|P_{B}\left(u_{n}\right)\right\|_{L^{2}}^{\alpha}+\|b\|_{L^{2}}\right]
\end{aligned}
$$

Then by (3.2), (3.4) and (2.1), there exist two positive constants $c_{2}, c_{3}$ such that

$$
\left\|u_{n}^{+}\right\| \leq c_{2}\left\|P_{B}\left(u_{n}\right)\right\|^{\alpha}+c_{3} .
$$

Observing that a similar result holds for $\left(u_{n}^{-}\right)$:

$$
\left\|u_{n}^{-}\right\| \leq c_{2}\left\|P_{B}\left(u_{n}\right)\right\|^{\alpha}+c_{3} .
$$

We conclude from (3.5) and (3.6) that the sequence $\left(u_{n}\right)$ is bounded if and only if the sequence $\left(P_{B}\left(u_{n}\right)\right)$ is bounded. Assume that $\left(P_{B}\left(u_{n}\right)\right)$ is not bounded, we can assume, by going to a subsequence if necessary, that $\left\|P_{B}\left(u_{n}\right)\right\| \rightarrow \infty$ as $n \rightarrow \infty$. Since $0 \leq \alpha<1$, we conclude by (3.5) and (3.6) that

$$
\frac{u_{n}^{+}}{\left\|P_{B}\left(u_{n}\right)\right\|} \rightarrow 0, \frac{u_{n}^{-}}{\left\|P_{B}\left(u_{n}\right)\right\|} \rightarrow 0 \text { as } n \rightarrow \infty .
$$

Therefore, we have

$$
y_{n}=\frac{u_{n}}{\left\|P_{B}\left(u_{n}\right)\right\|} \rightarrow y \in B, y \in B,|y|=1 \text { as } n \rightarrow \infty .
$$

It follows that

$$
\frac{\left|u_{n}^{0}\right|}{\left\|P_{B}\left(u_{n}\right)\right\|} \rightarrow 1 \text { as } n \rightarrow \infty .
$$

Consequently, by (3.5), (3.6) and (3.9), we can find a positive constant $c_{4}$ such that 


$$
\left\|u_{n}^{i}\right\| \leq c_{4}\left|u_{n}^{0}\right|^{\alpha}, i=+,-.
$$

Now, we apply the fact that $\left(\varphi\left(u_{n}+v_{n}\right)\right)$ is bounded to get

$$
\begin{aligned}
& \frac{\left\|u_{n}^{+}\right\|^{2}-\left\|u_{n}^{-}\right\|^{2}}{\left|u_{n}^{0}\right|^{2 \alpha}} \int_{0}^{T} \frac{H\left(t, u_{n}+v_{n}\right)}{\left|u_{n}^{0}\right|^{2 \alpha}} \mathrm{d} t \\
& +\int_{0}^{T} \frac{e(t) \cdot\left(u_{n}^{+}-u_{n}^{-}\right)}{\left|u_{n}^{0}\right|^{2 \alpha}} \mathrm{d} t \leq \frac{c_{5}}{\left|u_{n}^{0}\right|^{2 \alpha}}
\end{aligned}
$$

where $c_{3}$ is a positive constant. Using (3.10) and (3.11), we can find a constant $c_{6}$ satisfying

$$
\begin{aligned}
\int_{0}^{T} \frac{H\left(t, u_{n}^{0}\right)}{\left|u_{n}^{0}\right|^{2 \alpha}} \mathrm{d} t & =\int_{0}^{T} \frac{H\left(t, u_{n}+v_{n}\right)}{\left|u_{n}^{0}\right|^{2 \alpha}} \mathrm{d} t \\
& +\int_{0}^{T} \frac{H\left(t, u_{n}^{0}\right)-H\left(t, u_{n}+v_{n}\right)}{\left|u_{n}^{0}\right|^{2 \alpha}} \mathrm{d} t \\
& \leq c_{6}+\int_{0}^{T} \frac{H\left(t, u_{n}^{0}\right)-H\left(t, u_{n}+v_{n}\right)}{\left|u_{n}^{0}\right|^{2 \alpha}} \mathrm{d} t
\end{aligned}
$$

On the other hand, by the Mean Value Theorem and assumption $\left(H_{1}\right)$, we have

$$
\begin{aligned}
& \int_{0}^{T}\left[H\left(t, u_{n}^{0}\right)-H\left(u_{n}+v_{n}\right)\right] \mathrm{d} t \\
& =-\int_{0}^{T} H^{\prime}\left(t, u_{n}^{0}+\theta\left(u_{n}^{+}+u_{n}^{-}+v_{n}\right)\right) \cdot\left(u_{n}^{+}+u_{n}^{-}+v_{n}\right) \\
& \leq \int_{0}^{T}\left[a(t)\left|P_{B}\left(u_{n}^{0}+\theta\left(u_{n}^{+}+u_{n}^{-}\right)\right)\right|^{\alpha}+b(t)\right] \\
& \times\left|u_{n}^{+}+u_{n}^{-}+v_{n}\right| \mathrm{d} t \\
& \leq\left[\|a\|_{L^{1-\alpha}} \frac{2}{1-\alpha}\left\|P_{B}\left(u_{n}^{0}+\theta\left(u_{n}^{+}+u_{n}^{-}\right)\right)\right\|_{L^{2}}^{\alpha}\right. \\
& \left.+\|b\|{ }_{L^{2}}\right]\left\|u_{n}^{+}+u_{n}^{-}+v_{n}\right\|_{L^{2}} .
\end{aligned}
$$

By considering (3.13) and Sobolev's embedding $E \hookrightarrow L^{2}\left(0, T ; \mathbb{R}^{2 N}\right)$ we can find a constant $c_{7}>0$ such that

$$
\begin{aligned}
& \int_{0}^{T}\left[H\left(t, u_{n}^{0}\right)-H\left(u_{n}+v_{n}\right)\right] \mathrm{d} t \\
& \leq c_{7}\left[\left|u_{n}^{0}\right|^{\alpha}+\left|\left\|u_{n}^{+}\right\|\right|^{\alpha}+\left\|u_{n}^{-}\right\|^{\alpha}\right]\left[\left\|u_{n}^{+}\right\|+\left\|u_{n}^{-}\right\|+1\right]
\end{aligned}
$$

After combining (3.10), (3.12) and (3.14), we get

$$
\int_{0}^{T} \frac{H\left(t, u_{n}^{0}\right)}{\left|u_{n}^{0}\right|^{2 \alpha}} \mathrm{d} t \leq c_{8}
$$

for some positive constant $c_{8}$. However, the condition
(3.15) contradicts $\left(H_{2}\right)(i)$ because $\left|u_{n}^{0}\right| \rightarrow \infty$ as $n \rightarrow \infty$. Consequently, $\left(u_{n}\right)$ is bounded in $X$. Going if necessary to a subsequence, we can assume that $u_{n} \rightarrow u$, $u_{n}^{0} \rightarrow u^{0}$ and $v_{n} \rightarrow v$. Notice that

$$
\begin{aligned}
& Q\left(u_{n}^{+}-u^{+}\right)=\left(\varphi_{n}^{\prime}\left(u_{n}+v_{n}\right)-\varphi_{n}^{\prime}(u+v)\right)\left(u_{n}^{+}-u^{+}\right) \\
& -\int_{0}^{T}\left[H^{\prime}\left(t, u_{n}+v_{n}\right)-H^{\prime}(t, u+v)+e(t)\right] \\
& \cdot\left(u_{n}^{+}-u^{+}\right) \mathrm{d} t
\end{aligned}
$$

which implies that $u_{n}^{+} \rightarrow u^{+}$in E. Similarly, $u_{n}^{-} \rightarrow u^{-}$ in E. It follows that $\left(u_{n}, v_{n}\right) \rightarrow(u, v)$ in $X$ and $\varphi^{\prime}(u+v)=0$. So $\varphi$ satisfies the $(P S)_{c}^{*}$ condition for all $c \in \mathbb{R}$. The Lemma 3.1 is proved.

Now, let us prove that the functional $\varphi$ satisfies the conditions a), b) and c) of Theorem 2.1.

a) Let $(u, v) \in W \times V$. By using the Mean Value Theorem, assumptions $\left(H_{0}\right),\left(H_{1}\right)$ and (2.1), we have

$\varphi(u+v)=-\|u\|^{2}+\int_{0}^{T} H(t, u+v) \mathrm{d} t+\int_{0}^{T} e(t) \cdot u \mathrm{~d} t$

$=-\|u\|^{2}+\int_{0}^{T} H(t, v) \mathrm{d} t$

$+\int_{0}^{T} H^{\prime}(t, v+\theta u) \cdot u \mathrm{~d} t+\int_{0}^{T} e(t) \cdot u \mathrm{~d} t$

$\leq-\|u\|^{2}+\int_{0}^{T} H(t, v) \mathrm{d} t+\int_{0}^{T}\left[a(t)\left|P_{B}(u)\right|^{\alpha}\right.$

$+b(t)]|u| \mathrm{d} t+\int_{0}^{T} e(t) \cdot u \mathrm{~d} t$

$\leq-\|u\|^{2}+\int_{0}^{T} H(t, v) \mathrm{d} t+\int_{0}^{T}\left[a(t)\left\|P_{B}(u)\right\|^{\alpha}\right.$

$+b(t)]|u| \mathrm{d} t+\int_{0}^{T} e(t) \cdot u \mathrm{~d} t$

$\leq-\|u\|^{2}+\int_{0}^{T} H(t, v) \mathrm{d} t+\|u\|_{L^{2}}$

$\times\left[\left(\int_{0}^{T} a^{2}(t)|u|^{2 \alpha} \mathrm{d} t\right)^{\frac{1}{2}}+\|b\|_{L^{2}}\right]+\int_{0}^{T} e(t) \cdot u \mathrm{~d} t$

$\leq-\|u\|^{2}+\|u\|\left[c_{9}\|u\|^{\alpha}+c_{10}\right]+c_{11}$

where $c_{9}, c_{10}, c_{11}$ are three positive constants. Since $0 \leq \alpha<1$, then

$\varphi(u+v) \rightarrow-\infty$ as $u \in W,\|u\| \rightarrow \infty$ uniformly in $v \in V$.

b) Let $(u, v) \in Z \times V$, with $u=u^{+}+u^{0}$. By using the Mean Value Theorem, we get

$$
\begin{aligned}
& \varphi(u+v)=\left\|u^{+}\right\|^{2}+\int_{0}^{T} H\left(t, u^{+}+u^{0}+v\right) \mathrm{d} t \\
& +\int_{0}^{T} e(t) \cdot u^{+} \mathrm{d} t \\
& =\left\|u^{+}\right\|^{2}+\int_{0}^{T} H\left(t, u^{0}\right) \mathrm{d} t \\
& +\int_{0}^{T} H^{\prime}\left(t, u^{0}+\theta\left(u^{+}+v\right)\right) \cdot\left(u^{+}+v\right)+\int_{0}^{T} e(t) \cdot u^{+} \mathrm{d} t
\end{aligned}
$$


By assumption $\left(H_{1}\right)$ and (2.1), we can find a constant $C_{12}>0$ such that

$$
\begin{aligned}
& \left|\int_{0}^{T} H^{\prime}\left(t, u^{0}+\theta\left(u^{+}+v\right)\right) \cdot\left(u^{+}+v\right) \mathrm{d} t+\int_{0}^{T} e(t) \cdot u^{+} \mathrm{d} t\right| \\
& \leq \int_{0}^{T}\left[a(t)\left|P_{B}\left(t, u^{0}+\theta\left(u^{+}+v\right)\right)\right|^{\alpha}+b(t)\right] \\
& \times\left|u^{+}+v\right| \mathrm{d} t+\|e\|_{L^{2}}\left\|u^{+}\right\|_{L^{2}} \\
& \leq\left\|u^{+}+v\right\|_{L^{+}} \\
& \times\left(\left[\int_{0}^{T} a^{2}(t)\left(\left|u^{0}\right|+\left|u^{+}\right|\right)^{2 \alpha}\right]^{\frac{1}{2}}+\|b\|_{L^{2}}+\|e\|_{L^{2}}\right) \\
& \leq c_{12}\left(\left\|u^{+}\right\|+1\right)\left[\left|u^{0}\right|^{\alpha}+\left\|u^{+}\right\|^{\alpha}+1\right]
\end{aligned}
$$

Therefore, by using (3.19) and (3.20) we obtain

$$
\begin{aligned}
\varphi(u+v) & \geq\left\|u^{+}\right\|^{2}+\int_{0}^{T} H\left(t, u^{0}\right) \mathrm{d} t \\
& -c_{12}\left(\left\|u^{+}\right\|+1\right)\left[\left|u^{0}\right|^{\alpha}+\left\|u^{+}\right\|^{\alpha}+1\right] .
\end{aligned}
$$

Now let $d>\frac{c_{12}^{2}}{2}$. By assumption $\left(H_{2}\right)(i)$, there exists a constant $C_{13}>0$ such that

$$
\int_{0}^{T} H\left(t, u^{0}\right) \mathrm{d} t \geq \mathrm{d}\left|u^{0}\right|^{2 \alpha}-c_{13}
$$

So by (3.21) and (3.22), we have

$$
\begin{aligned}
\varphi(u+v) & \geq\left\|u^{+}\right\|^{2}+d\left|u^{0}\right|^{2 \alpha}-c_{13} \\
& -c_{12}\left(\left\|u^{+}\right\|+1\right)\left[\left|u^{0}\right|^{\alpha}+\left\|u^{+}\right\|^{\alpha}+1\right] \\
& \geq \frac{1}{2}\left\|u^{+}\right\|^{2}-c_{12}\left[\left\|u^{+}\right\|^{\alpha+1}-\left\|u^{+}\right\|-\left\|u^{+}\right\|^{\alpha}\right] \\
& +\frac{1}{2}\left[\left\|u^{+}\right\|-c_{12}\left|u^{0}\right|^{\alpha}\right]^{2}+\left[d-\frac{c_{12}^{2}}{2}\right]\left|u^{0}\right|^{2 \alpha} \\
& -c_{12}\left|u^{0}\right|^{\alpha}-c_{12}-c_{13} .
\end{aligned}
$$

Since $d>\frac{c_{12}^{2}}{2}$ and $0 \leq \alpha<1$, then

$$
\varphi(u+v) \rightarrow \infty \text { as } u \in Z,\|u\| \rightarrow \infty \text {, uniformly in } v \in V \text {. }
$$

Hence by Lemma 3.1 and properties (3.18), (3.24), we deduce that the functional $\varphi$ satisfies all the assumptions of Theorem 2.1. Therefore the Hamiltonian system $(\mathcal{H})$ possesses at least $(p+1) T-$ periodic solutions geometrically distinct. The proof of Theorem 1.1 is complete.

Proof of Theorem 1.2. Assume $\left(H_{0}\right),\left(H_{1}\right)$ and $\left(H_{4}\right)(i)$ hold. The following lemma will be needed for the study of the geometry of the functional $\varphi$.

Lemma 3.2. There exist a non-increasing positive function $\theta \in C(] 0, \infty\left[, \mathbb{R}^{+}\right)$and a positive constant $c_{0}$ satisfying the following conditions:

i) $\theta(s) \rightarrow 0, \theta(s) s \rightarrow+\infty$ as $s \rightarrow+\infty$,

ii) $\left\|H^{\prime}(t, u)\right\|_{L^{2}} \leq c_{0}\left[\theta\left(\left\|P_{B}(u)\right\|\right)\left\|P_{B}(u)\right\|+1\right], \forall u \in E$,

iii) $\frac{1}{\left[\theta\left(\left|u^{0}\right|\right)\left|u^{0}\right|\right]^{2}} \int_{0}^{T} H\left(t, u^{0}\right) \mathrm{d} t \rightarrow+\infty$ as $\left|u^{0}\right| \rightarrow+\infty$.

Proof: For $u \in E$, let

$$
A=\left\{t \in[0, T]:\left|P_{B}(u)(t)\right| \geq \|\left. P_{B}(u)\right|^{\mid \frac{1}{2}}\right\} .
$$

By $\left(H_{3}\right)$, we have

$$
\begin{aligned}
& \left\|H^{\prime}(t, u)\right\|_{L^{2}} \leq\left[\int_{0}^{T}\left[a \omega\left(\left|P_{B}(u)(t)\right|\right)\left|P_{B}(u)(t)\right|+g(t)\right]^{2} \mathrm{~d} t\right]^{\frac{1}{2}} \\
& \leq\left[\int_{0}^{T}\left[a \omega\left(\left|P_{B}(u)(t)\right|\right)\left|P_{B}(u)(t)\right|\right]^{2} \mathrm{~d} t\right]^{\frac{1}{2}}+\|g\|_{L^{2}} \\
& \leq a\left[\int_{A} \omega^{2}\left(P_{B}(u)(t)\right)\left|P_{B}(u)(t)\right|^{2} \mathrm{~d} t\right.
\end{aligned}
$$$$
\left.+\int_{[0, T]-A} \omega^{2}\left(P_{B}(u)(t)\right)\left|P_{B}(u)(t)\right|^{2} \mathrm{~d} t\right]^{\frac{1}{2}}+\|g\|_{L^{2}}
$$$$
\leq a\left[\int_{A} \omega^{2}\left(\left\|P_{B}(u)\right\|^{\frac{1}{2}}\right)\left|P_{B}(u)(t)\right|^{2} \mathrm{~d} t\right.
$$$$
\left.+T \sup _{s \geq 0} \omega^{2}(s)\left\|P_{B}(u)\right\|\right]^{\frac{1}{2}}+\|g\|_{L^{2}} \text {. }
$$

So, by (2.1) there exists a positive constant $c_{0}$ such that

$$
\begin{aligned}
& \left\|H^{\prime}(t, u)\right\|_{L^{2}} \\
& \leq c_{0}\left(\left[\omega^{2}\left(\left\|P_{B}(u)\right\|^{\frac{1}{2}}\right)\left\|P_{B}(u)\right\|^{2}+\left\|P_{B}(u)\right\|^{\frac{1}{2}}+1\right) .\right.
\end{aligned}
$$

Take

$$
\theta(s)=\left[\omega^{2}\left(s^{\frac{1}{2}}\right)+\frac{1}{s}\right]^{\frac{1}{2}}, s>0,
$$

then $\theta$ satisfies (ii) and it is clear to see that $\theta$ satisfies (i).

Next, let us define

$$
\rho=\liminf _{s \rightarrow \infty} \frac{\omega^{2}(s)}{\omega^{2}\left(s^{\frac{1}{2}}\right)} .
$$

By $\left(H_{4}\right)(i)$, for any $\gamma>0$, there exists a positive 
constant $C_{14}$ such that

$$
\int_{0}^{T} H(t, x) \mathrm{d} t \geq \gamma[\omega(|x|)|x|]^{2}-c_{14} .
$$

which implies that for $u_{0} \in B, u^{0} \neq 0$,

$$
\frac{\int_{0}^{T} H\left(t, u^{0}\right) \mathrm{d} t}{\left[\theta\left(\left|u^{0}\right|\right)\left|u^{0}\right|\right]^{2}} \geq \frac{\gamma\left[\omega\left(\left|u^{0}\right|\right)\left|u^{0}\right|\right]^{2}-c_{14}}{\omega^{2}\left(\left|u^{0}\right|^{\frac{1}{2}}\right)\left|u^{0}\right|^{2}+\left|u^{0}\right|}
$$

By the definition of $\rho$, there exists $R>0$ such that for all $s \geq R$

$$
\frac{\omega^{2}(s) s^{2}}{\omega^{2}\left(s^{\frac{1}{2}}\right) s^{2}+s} \geq \frac{\rho}{2}
$$

Therefore

$$
\frac{\int_{0}^{T} H\left(t, u^{0}\right) \mathrm{d} t}{\left[\theta\left(\left|u^{0}\right|\right)\left|u^{0}\right|\right]^{2}} \geq \frac{\gamma \rho}{2 T}-\frac{c_{14}}{\omega^{2}\left(\left|u^{0}\right|^{\frac{1}{2}}\right)\left|u^{0}\right|^{2}+\left|u^{0}\right|}
$$

as $\left|u^{0}\right| \geq R$ and then

$$
\lim _{\left|u^{0}\right| \rightarrow \infty} \frac{\int_{0}^{T} H\left(t, u^{0}\right) \mathrm{d} t}{\left[\theta\left(\left|u^{0}\right|\right)\left|u^{0}\right|\right]^{2}} \geq \frac{\gamma \rho}{2 T} .
$$

Since $\gamma$ is arbitrary choosen, condition (iii) holds.

Now, let us prove the Palais-Smale condition.

Lemma 3.3. For all level $c \in \mathbb{R}$, the functional $\varphi$ satisfies the $(P S)_{c}^{*}$ condition with respect to the sequence $\left(X_{n}\right)_{n \in \mathbb{N}}$.

Proof. Let $\left(u_{n}, v_{n}\right)_{n \in \mathbb{N}}$ be a sequence in $X$ such that for all $n \in \mathbb{N},\left(u_{n}, v_{n}\right) \in X_{n}$ and

$$
\varphi\left(u_{n}+v_{n}\right) \rightarrow c \text { and } \varphi_{n}^{\prime}\left(u_{n}+v_{n}\right) \rightarrow 0 \text { as } n \rightarrow \infty \text {. }
$$

Set $u_{n}=u_{n}^{+}+u_{n}^{-}+u_{n}^{0}$ and $\tilde{u}_{n}=u_{n}^{+}+u_{n}^{-}$. By Hölder's inequality, (2.1) and Lemma 2.2(ii), we get a positive constant $C_{15}$ such that

$$
\begin{aligned}
& \left|\int_{0}^{T}\left(H^{\prime}\left(t, u_{n}+v_{n}\right)+e(t)\right) \cdot\left(u_{n}^{+}-u_{n}^{-}\right) \mathrm{d} t\right| \\
& \leq\left\|u_{n}^{+}-u_{n}^{-}\right\|_{L^{2}}\left(\left[\int_{0}^{T}\left|H^{\prime}\left(t, u_{n}+v_{n}\right)\right|^{2}\right]^{\frac{1}{2}}+\|e\|_{L^{2}}\right) \\
& \leq c_{15}\left\|\tilde{u}_{n}\right\|\left[\theta\left(\left\|P_{B}\left(u_{n}\right)\right\|\right)\left\|P_{B}\left(u_{n}\right)\right\|+1\right] .
\end{aligned}
$$

Thus, for $n$ large enough

$$
\begin{aligned}
\left\|\tilde{u}_{n}\right\| & \geq \varphi^{\prime}\left(u_{n}+v_{n}\right)\left(u_{n}^{+}-u_{n}^{-}\right) \\
& \geq 2\left\|\tilde{u}_{n}\right\|^{2}-c_{15}\left\|\tilde{u}_{n}\right\|\left[\theta\left(\left\|P_{B}\left(u_{n}\right)\right\|\right)\left\|P_{B}\left(u_{n}\right)\right\|+1\right] .
\end{aligned}
$$

So there exists a positive constant $c_{16}$ such that

$$
\left\|\tilde{u}_{n}\right\| \leq c_{16}\left[\theta\left(\left\|P_{B}\left(u_{n}\right)\right\|\right)\left\|P_{B}\left(u_{n}\right)\right\|+1\right] .
$$

By (3.33) and the properties (i) of $\theta$, we deduce that $\left(\left\|u_{n}\right\|\right)$ is bounded if and only $\left(\left\|P_{B}\left(u_{n}\right)\right\|\right)$ is bounded.

Now, since $\theta$ is nonincreasing and $\|u\| \geq\left\|P_{B}\left(u_{n}\right)\right\|$ $\geq \max \left(\left|u^{0}\right|,\left\|P_{B}(\tilde{u})\right\|\right)$, we get

$$
\theta(\|u\|) \leq \min \left(\theta\left(\left|u^{0}\right|\right), \theta\left(\left\|P_{B}(\tilde{u})\right\|\right)\right)
$$

Combining (3.32) and (3.34), yields for $n$ large enough

$$
\begin{aligned}
& \left\|\tilde{u}_{n}\right\| \geq 2\left\|\tilde{u}_{n}\right\|^{2} \\
& -c_{15}\left\|\tilde{u}_{n}\right\|\left[\theta\left(\left\|P_{B}\left(\tilde{u}_{n}\right)\right\|\right)\left\|P_{B}\left(\tilde{u}_{n}\right)\right\|+\theta\left(\left|u_{n}^{0}\right|\right)\left|u_{n}^{0}\right|+1\right]
\end{aligned}
$$

which implies

$$
c_{15} \theta\left(\left|u_{n}^{0}\right|\right)\left|u_{n}^{0}\right| \geq\left\|\tilde{u}_{n}\right\|\left[2-c_{15} \theta\left(\left\|P_{B}\left(\tilde{u}_{n}\right)\right\|\right)\right]-c_{15}-1 .
$$

Assume that $\left(\left\|P_{B}\left(\tilde{u}_{n}\right)\right\|\right)$ is unbounded, then by going to a subsequence, if necessary, we can assume that $\left\|P_{B}\left(\tilde{u}_{n}\right)\right\| \rightarrow \infty$ as $n \rightarrow \infty$. Since $\theta(s) \rightarrow 0$ as $s \rightarrow \infty$, we deduce from (3.35) that there exists a positive constant $C_{17}$ such that

$$
\left\|\tilde{u}_{n}\right\| \leq c_{17} \theta\left(\left|u_{n}^{0}\right|\right)\left|u_{n}^{0}\right|
$$

for $n$ large enough. Since the map $s \rightarrow \theta(s) s$ is continuous in $[0, \infty]$ and goes to $+\infty$ as $s \rightarrow \infty$, then $\left|u_{n}^{0}\right| \rightarrow \infty$ as $n \rightarrow \infty$.

Now, by the Mean Value Theorem, Hölder's inequality and Lemma 3.2(ii), we get

$$
\begin{aligned}
& \left|\int_{0}^{T}\left(H\left(t, u_{n}+v_{n}\right)-H\left(t, u_{n}^{0}\right)\right) \mathrm{d} t\right| \\
& \left|\int_{0}^{T} \int_{0}^{1} H^{\prime}\left(t, u_{n}^{0}+s\left(\tilde{u}_{n}+v_{n}\right)\right) \cdot\left(\tilde{u}=+v_{n}\right) \mathrm{d} s \mathrm{~d} t\right| \\
& \leq\left\|\tilde{u}_{n}+v_{n}\right\|_{L^{2}} \int_{0}^{1}\left(\int_{0}^{T}\left|H^{\prime}\left(t, u_{n}^{0}+s\left(\tilde{u}_{n}+v_{n}\right)\right)\right|^{2} \mathrm{~d} t\right)^{\frac{1}{2}} \mathrm{~d} s \\
& \leq c_{0}\left\|\tilde{u}_{n}+v_{n}\right\|_{L^{2}} \int_{0}^{1}\left[\theta\left(\left\|u_{n}^{0}+s P_{B}\left(\tilde{u}_{n}\right)\right\|\right)\left\|u_{n}^{0}+s P_{B}\left(\tilde{u}_{n}\right)\right\|+1\right] \mathrm{d} s .
\end{aligned}
$$

Since ||$u_{n}^{0}+s P_{B}\left(\tilde{u}_{n}\right)|\geq| u_{n}^{0} \mid \quad$ for all $s \in[0,1]$, we dedu-ce from (2.1), (3.36) and (3.37) that there exists a positive constant $C_{18}$ such that

$$
\begin{aligned}
& {\left[\int_{0}^{T}\left(H\left(t, u_{n}+v_{n}\right)-H\left(t, u_{n}^{0}\right)\right) \mathrm{d} t\right)} \\
& \leq c_{0}||\left|\tilde{u}_{n}+v_{n} \|\right|_{L^{2}}\left[\theta\left(\left|u_{n}^{0}\right|\right)\left|u_{n}^{0}\right|+\theta\left(\left|u_{n}^{0}\right|\right)\left\|P_{B}\left(\tilde{u}_{n}\right)\right\|+1\right] \\
& \leq c_{18}\left[\left[\theta\left(\left|u_{n}^{0}\right|\right)\left|u_{n}^{0}\right|\right]^{2}+\theta\left(\left|u_{n}^{0}\right|\right)\left[\theta\left(\left|u_{n}^{0}\right|\right)\left|u_{n}^{0}\right|\right]^{2}\right. \\
& \left.+\theta\left(\left|u_{n}^{0}\right|\right)\left|u_{n}^{0}\right|+1\right],
\end{aligned}
$$

which with (2.1) and (3.36) imply that there exists a positive constant $C_{19}$ such that 


$$
\begin{aligned}
& \varphi\left(u_{n}+v_{n}\right)=\left\|u_{n}^{+}\right\|^{2}-\left\|u_{n}^{-}\right\|^{2}+\int_{0}^{T} H\left(t, u_{n}^{0}\right) \mathrm{d} t \\
& +\int_{0}^{T}\left(H\left(t, u_{n}+v_{n}\right)-H\left(t, u_{n}^{0}\right)\right) \mathrm{d} t+\int_{0}^{T} e(t) \cdot \tilde{u}_{n} \mathrm{~d} t \\
& \geq+\int_{0}^{T} H\left(t, u_{n}^{0}\right) \mathrm{d} t-c_{19}\left[\left[\theta\left(\left|u_{n}^{0}\right|\right)\left|u_{n}^{0}\right|\right]^{2}\right. \\
& \left.+\theta\left(\left|u_{n}^{0}\right|\right)\left[\theta\left(\left|u_{n}^{0}\right|\right)\left|u_{n}^{0}\right|\right]^{2}+\theta\left(\left|u_{n}^{0}\right|\right)\left|u_{n}^{0}\right|+1\right] \\
& \geq c_{19}\left[\theta\left(\left|u_{n}^{0}\right|\right)\left|u_{n}^{0}\right|\right]^{2}\left(-1-\theta\left(\left|u_{n}^{0}\right|\right)-\frac{1}{\theta\left(\left|u_{n}^{0}\right|\right)\left|u_{n}^{0}\right|}\right. \\
& \left.-\frac{1}{\left[\theta\left(\left|u_{n}^{0}\right|\right)\left|u_{n}^{0}\right|\right]^{2}}+\frac{\int_{0}^{T} H\left(t, u_{n}^{0}\right) \mathrm{d} t}{c_{19}\left[\theta\left(\left|u_{n}^{0}\right|\right)\left|u_{n}^{0}\right|\right]^{2}}\right)
\end{aligned}
$$

which, with Lemma 3.2 (iii), imply that $\varphi\left(u_{n}+v_{n}\right) \rightarrow \infty$ as $n \rightarrow \infty$. This contradicts the boundedness of $\left(\varphi\left(u_{n}+v_{n}\right)\right)$. So $\left(\left\|P_{B}\left(\tilde{u}_{n}\right)\right\|\right)$ is bounded.

Assume that $\left(\left|u_{n}^{0}\right|\right)$ is unbounded, then up to a subsequence, if necessary, we can assume that $\left|u_{n}^{0}\right| \rightarrow \infty$ as $n \rightarrow \infty$. As in (3.38), and using (2.1), (3.34) and the fact that $\theta(s) \rightarrow 0$ as $s \rightarrow \infty$, we can find a constant $c_{21}>0$ such that

$$
\begin{aligned}
& \left|\int_{0}^{T}\left(H\left(t, u_{n}+v_{n}\right)-H\left(t, u_{n}^{0}\right)\right) \mathrm{d} t\right| \\
& \leq c_{0}\left\|\tilde{u}_{n}+v_{n}\right\|_{L^{2}}\left[\theta\left(\left|u_{n}^{0}\right|\right)\left|u_{n}^{0}\right|+\theta\left(\left|u_{n}^{0}\right|\right)\left\|P_{B}\left(\tilde{u}_{n}\right)\right\|+1\right] \\
& \leq c_{21}\left\|\tilde{u}_{n}+v_{n}\right\|\left[\theta\left(\left|u_{n}^{0}\right|\right)\left|u_{n}^{0}\right|+1\right] .
\end{aligned}
$$

Now, since $\theta(s) \rightarrow 0$ as $s \rightarrow \infty$, then combining (3.33) and (3.34) yields

$$
\begin{aligned}
\left\|\tilde{u}_{n}+v_{n}\right\| & \leq\left[\theta\left(\left|u_{n}^{0}\right|\right)\left|u_{n}^{0}\right|+\theta\left(\left|u_{n}^{0}\right|\right)\left\|P_{B}\left(\tilde{u}_{n}\right)\right\|+1\right]+\left|v_{n}\right| \\
& \leq c_{22}\left[\theta\left(\left|u_{n}^{0}\right|\right)\left|u_{n}^{0}\right|+1\right]
\end{aligned}
$$

for a positive constant $c_{22}$. Therefore there exists a positive constant $C_{23}$ such that

$$
\begin{aligned}
& \left|\int_{0}^{T}\left(H\left(t, u_{n}+v_{n}\right)-H\left(t, u_{n}^{0}\right)+e(t) \cdot \tilde{u}_{n}\right) \mathrm{d} t\right| \\
& \leq c_{23}\left[\theta\left(\left|u_{n}^{0}\right|\right)\left|u_{n}^{0}\right|+1\right]^{2} .
\end{aligned}
$$

We deduce from (3.41) and (3.42) that there exists a constant $c_{24}>0$ such that

$$
\begin{aligned}
\varphi\left(u_{n}+v_{n}\right) & \geq-c_{24}\left[\theta\left(\left|u_{n}^{0}\right|\right)\left|u_{n}^{0}\right|+1\right]^{2}+\int_{0}^{T} H\left(t, u_{n}^{0}\right) \mathrm{d} t \\
& \geq\left[\theta\left(\left|u_{n}^{0}\right|\right)\left|u_{n}^{0}\right|+1\right]^{2}\left[-c_{24}+\frac{\int_{0}^{T} H\left(t, u_{n}^{0}\right) \mathrm{d} t}{\left[\theta\left(\left|u_{n}^{0}\right|\right)\left|u_{n}^{0}\right|+1\right]^{2}}\right]
\end{aligned}
$$

which implies by Lemma 3.2 (iii) that $\varphi\left(u_{n}+v_{n}\right) \rightarrow+\infty$ as $n \rightarrow \infty$. This contradicts the boundedness of $\left(\varphi\left(u_{n}+v_{n}\right)\right)$. Then $\left(\left|u_{n}^{0}\right|\right)$ is also bounded and therefore $\left(\left\|u_{n}\right\|\right)$ is bounded. By a standard argument, we con-clude that $\left(u_{n}\right)$ possesses a convergent subsequence. The proof of Lemma 3.3 is complete.

Now, let $(u, v)=\left(u^{0}+u^{+}, v\right) \in Z \times V$, then as in (3.38) there exists a positive constant $c_{25}$ such that

$$
\begin{aligned}
& \left|\int_{0}^{T}\left(H\left(t, u_{n}+v_{n}\right)-H\left(t, u_{n}^{0}\right)+e(t) \cdot \tilde{u}_{n}\right) \mathrm{d} t\right| \\
& \leq c_{25}\left[\mid u^{+} \|+1\right]\left[\theta\left(\left|u^{0}\right|\right)\left|u^{0}\right|+\theta\left(\left|u^{0}\right|\right)|| P_{B}\left(u^{+}\right) \|+1\right] .
\end{aligned}
$$

So, we have for a positive constant $c_{26}$

$$
\begin{aligned}
\varphi(u+v) & \geq\left\|u^{+}\right\|^{2}-c_{26}\left\|u^{+}\right\|\left[\theta\left(\left|u^{0}\right|\right)\left|u^{0}\right|\right. \\
& \left.+\theta\left(\left|u^{0}\right|\right)\left\|u^{+}\right\|+1\right]-c_{26}+\int_{0}^{T} H\left(t, u_{n}^{0}\right) \mathrm{d} t .
\end{aligned}
$$

Let $0<\epsilon<1$, we have

$$
c_{26} \theta\left(\left|u^{0}\right|\right)\left|u^{0}\right|\left\|u^{+}\right\| \leq \frac{c_{26}^{2}}{\epsilon^{2}}\left[\theta\left(\left|u_{n}^{0}\right|\right)\left|u_{n}^{0}\right|\right]^{2}+\epsilon^{2}\left\|u^{+}\right\|^{2} \text {. }
$$

By combining (3.44) and (3.45), we get

$$
\begin{aligned}
\varphi(u+v) \geq & {\left[1-\epsilon^{2}-c_{26} \theta\left(\left|u^{0}\right|\right)\right]\left\|u^{+}\right\|^{2}-c_{26}\left\|u^{+}\right\| } \\
& +\left[\theta\left(\left|u_{n}^{0}\right|\right)\left|u_{n}^{0}\right|\right]^{2}\left[-\frac{c_{26}^{2}}{\epsilon^{2}}+\frac{\int_{0}^{T} H\left(t, u_{n}^{0}\right) \mathrm{d} t}{\left[\theta\left(\left|u_{n}^{0}\right|\right)\left|u_{n}^{0}\right|+1\right]^{2}}\right]
\end{aligned}
$$

which implies that

$\varphi(u+v) \rightarrow+\infty$ as $u \in Z,\|u\| \rightarrow \infty$, uniformly in $v \in V$.

On the other hand, let $b \in B,|b|>0$. By the Mean Value Theorem, we have for $u \in W=E^{-}$

$$
\begin{aligned}
& \left|\int_{0}^{T}(H(t, u+v)-H(t, b)) \mathrm{d} t\right| \\
& =\left|\int_{0}^{T} \int_{0}^{1} H^{\prime}(t, b+s(u+v-b)) \cdot(u+v-b) \mathrm{d} s \mathrm{~d} t\right| \\
& \leq\|u+v-b\|_{L^{2}} \int_{0}^{1}\left[\left.\int_{0}^{T} H^{\prime}(t, b+s(u+v-b))\right|^{2} \mathrm{~d} t\right]^{\frac{1}{2}} \mathrm{~d} s \\
& \leq\|u+v-b\|_{L^{2}} \int_{0}^{1}\left[\int _ { 0 } ^ { T } \left(a \omega\left(\left|b+s\left(P_{B}(u)-b\right)\right|\right)\right.\right. \\
& \left.\left.\times\left|b+s\left(P_{B}(u)-b\right)\right|+g(t)\right)^{2} \mathrm{~d} t\right]^{\frac{1}{2}} \mathrm{~d} s \\
& \leq\|u+v-b\|_{L^{2}}\left(a \int _ { 0 } ^ { 1 } \left[\int_{0}^{T}\left(\omega\left(\left|b+s\left(P_{B}(u)-b\right)\right|\right)\right]\right.\right. \\
& \left.\left.\times\left|b+s\left(P_{B}(u)-b\right)\right|\right)^{2} \mathrm{~d} t\right]^{\frac{1}{2}} \mathrm{~d} s+\|g\|_{L^{2}}
\end{aligned}
$$


Take for $s \in[0,1]$,

$$
A(s)=\left\{t \in[0,1]:\left|b+s\left(P_{B}(u)-b\right)\right| \geq|b|\right\} .
$$

By a similar calculation as in the proof of Lemma 3.2, we get for some positive constants $c_{27}$ and $c(b)$

$$
\begin{aligned}
& \left|\int_{0}^{T}(H(t, u+v)-H(t, b)+e(t) \cdot u) \mathrm{d} t\right| \\
& \leq c_{27} \omega(|b|) \mid u \|^{2}+c(b)(\|u\|+1)
\end{aligned}
$$

which implies that

$$
\begin{aligned}
\varphi(u+v) & \leq-\|u\|^{2}+c_{27} \omega(|b|)\|u\|^{2} \\
& +c(b)(\|u\|+1)+\int_{0}^{T} H(t, b) \mathrm{d} t .
\end{aligned}
$$

Since $\theta(s) \rightarrow 0$ ass $\rightarrow \infty$, there exists $|b|>0$ such that $c_{27} \omega(|b|) \leq \frac{1}{2}$, which implies that

$$
\varphi(u+v) \leq-\frac{1}{2}\|u\|^{2}+c(b)(\|u\|+1)+\int_{0}^{T} H(t, b) \mathrm{d} t .
$$

So we have

$$
\begin{array}{r}
\varphi(u+v) \rightarrow-\infty \text { as } u \in W,\|u\| \rightarrow \infty, \text { uniformly in } \\
v \in \in V . \text { (3.50) }
\end{array}
$$

Thus, Lemma 3.3 and properties (3.46), (3.50) imply that the functional $\varphi$ satisfies all the assumptions of the Generalized Saddle Point Theorem. Therefore the Hamiltonian system $(\mathcal{H})$ possesses at least $(p+1) T-$ periodic solutions geometrically distinct. The proof of Theorem 1.2 is complete.

\section{References}

[1] T. An, "Periodic Solutions of Superlinear Autonomous Hamiltonian Systems with Prescribed Period," Journal of
Mathematical Analysis Application, Vol. 323, No. 15, 2006, pp. 854-863. doi:10.1016/j.jmaa.2005.11.004

[2] P. L. Felmer, "Periodic Solutions of Superquadratic Hamiltonian Systems," Journal of Differential Equation, Vol. 102, No. 1, 1993, pp. 188-207. doi:10.1006/jdeq.1993.1027

[3] Z. Q. Ou and C. L. Tang, "Periodic and Subharmonic Solutions for a Class of Superquadratic Hamiltonian Systems," Nonlinear Analysis, Vol. 58, No. 3-4, 2004, pp. 245-258. doi:10.1016/j.na.2004.03.029

[4] C. L. Tang and X. P. Wu, "Periodic Solutions for Second Order Systems with Not Uniformly Coercive Potential," Journal of Mathematical Analysis Application, Vol. 259, No. 2, 2001, pp. 386-397. doi:10.1006/jmaa.2000.7401

[5] M. Timoumi, "Periodic Solutions for Noncoercive Hamiltonian Systems," Demonstratio Mathematica, Vol. 35, No. 4, 2002, pp. 899-913.

[6] K. C. Chang, "On the Periodic Nonlinearity and the Multiplicity of Solutions," Nonlinear Analysis, Vol. 13, No. 5 1989, pp. 527-537. doi:10.1016/0362-546X(89)90062-X

[7] S. X. Chen, X. Wu and F. Zhao, "New Existence and Multiplicity Theorems of Periodic Solutions for NonAutonomous Second Order Hamiltonian Systems," Mathematical and Computer Modeling, Vol. 46, No. 3-4, 2007, pp. 550-556. doi:10.1016/j.mcm.2006.11.019

[8] I. Ekeland and J. M. Lasry, "On the Number of Periodic Trajectories for a Hamiltonian Flow on a Convex Energy Surface," Annals of Mathematics, Vol. 112, No. 2, 1980, pp. 283-319. doi:10.2307/1971148

[9] M. Timoumi, "On the Multiplicity of Periodic Solutions of a Hamiltonian System," Demonstratio Mathematica, Vol. 35, No. 4, 2002, pp. 899-913.

[10] G. Fournier, D. Lupo, M. Ramos and M. Willem, "Limit Relative Category and Critical Point Theory," Dynamics Reported, Vol. 3, 1994, pp. 1-24. 